\title{
Performance Analysis for Adaptive MIMO SVD Transmission in a Cellular System
}

\author{
Peter J. Smith ${ }^{\star} \quad$ Mansoor Shafi ${ }^{\dagger}$ Lee M. Garth ${ }^{\star}$ \\ * Department of Electrical and Computer Engineering, University of Canterbury, Christchurch, New Zealand \\ ${ }^{\dagger}$ Telecom New Zealand Limited, Wellington, New Zealand
}

\begin{abstract}
In this paper we consider the performance of an adaptive MIMO SVD (singular value decomposition) transmission scheme operating in a cellular environment. In particular we consider the impact of inter-cell interference on the performance of an adaptive MIMO system. Inter-cell interference is always present in a cellular system. However, its value may be controlled by the type of cellular layout, i.e., the number of sectors and the frequency reuse pattern. In this paper we consider a number of cellular layouts and consider the impact of the resulting SINR on the constellation sizes that can be supported, the BER, etc. The primary metric used for our performance analysis is the error free transmission rate, and this is derived for the adaptive MIMO SVD system. For the cellular scenarios considered, we find that the effect of interference is considerable and the performance of the adaptive MIMO SVD scheme is only marginally better than that provided by conventional diversity methods.
\end{abstract}

\section{INTRODUCTION}

The pioneering work of [1], [2] has resulted in immense interest in MIMO systems. They offer the promise of large system capacities, and thus are being considered for fourth generation wireless systems. However, the majority of work in this area has focussed on single-user MIMO systems, and recent results [3] suggest that the promised rates may not be available in cellular systems. Hence, in this paper we study the performance of an adaptive MIMO SVD transmission scheme in a cellular environment and make the following contributions:

- We consider the error free rate of the system, a performance metric that is useful in comparing both fixed and adaptive transmission schemes with varying constellation sizes. The error free rate can also be defined as the mean number of correctly received bits per symbol period.

- The metric is derived in the case of an adaptive MIMO SVD transmission scheme using uncoded transmission in an independent and identically distributed (i.i.d.) Rayleigh channel and is verified by simulation.

- We show that the performance of adaptive MIMO SVD is heavily degraded under low SINR conditions. As a result, the error free rate, averaged over the cellular interference, is only marginally better than that provided by conventional diversity methods.

- Our results provide new insights in understanding the performance of adaptive MIMO systems. For example, at low BERs, increasing the variance of the SINR distribution gives better performance. We also quantify the trade off between the BER and constellation size.

- We compare the performance of the adaptive scheme with that of a fixed modulation system employing MIMO-
MMSE receivers and demonstrate the improvements offered by the SVD approach.

- We show that the improvements offered by the adaptive SVD approach are at the expense of suspending transmission, and these periods of no communication occur with high probability at SINR values below $5 \mathrm{~dB}$.

Finally, our analytical method for the Rayleigh channel can be extended to derive results for semi-correlated Rayleigh and i.i.d. Ricean channels, but this is beyond the scope of the paper.

The layout of the paper is as follows. In Sec. II we describe the cellular layouts considered and define the adaptive MIMO SVD transmission scheme. In Sec. III we derive the error free rate of the system, and in Sec. IV we give our simulation results. Finally, some conclusions are given in Sec. V.

\section{BACKGROUND}

Here we consider a single MIMO system with perfect channel state information (CSI) at both the transmitter and receiver, operating in a cellular environment. The effect of the interference on the MIMO system is catered for by a simple scaling of the additive noise power. Hence, we assume that the interference is Gaussian and unknown at the receiver.

\section{A. Cellular Layouts}

In order to study the impact of inter-cell interference, we consider a system based on an industry standard [4] 19-cell cluster of hexagonal cells as shown in Fig. 1. Each of the cells employs either 3,6 or 12 sectors as shown in Fig. 2. Furthermore, among neighbouring cells one may either use the same frequency $F=1$, or employ a reuse pattern of 3 , $F=1 / 3$, as discussed in [3]. The user is connected to the base station with the strongest signal. All other base stations are then deemed as interferers. By randomly placing users in the desired and surrounding cells, and by assuming appropriate values for the path loss exponent and log normal shadowing, one can find a signal-to-interference and noise ratio (SINR), denoted $\Gamma$. The SINR can now be generated by the procedure in [3]. The resulting SINR cumulative distributions are shown in Fig. 3. The different scenarios are referred to as R1S3, R1S6, R1S12, R3S3, R3S6, R3S12 (the notation R1S3 stands for a reuse of 1 with 3 sectors).

\section{B. Channel Model and SVD Transmission}

We model the channel as a flat fading i.i.d. Rayleigh channel. For a MIMO system with $n_{T}$ transmit and $n_{R}$ receive 


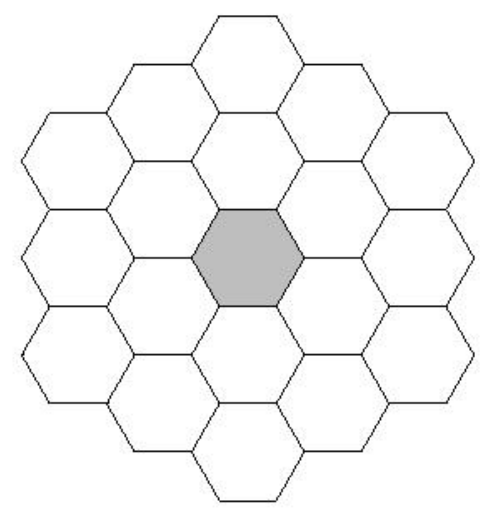

1: Cluster of 19 cells with the desired cell shaded.
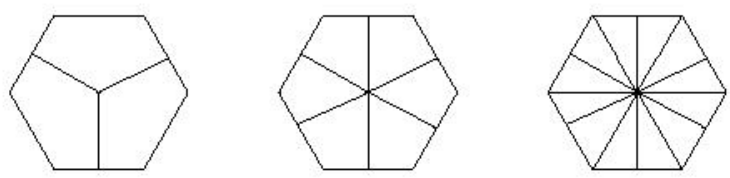

2: A single cell split into 3,6 and 12 sectors.

antennas the received signal can be written

$$
r=H s+n
$$

where $r$ is the $n_{R} \times 1$ received signal vector, and $s$ is the complex $n_{T} \times 1$ transmitted signal vector with entries having unit magnitude variance. The matrix $H$ is an $n_{R} \times n_{T}$ complex channel gain matrix with entries also having unit magnitude variance. The AWGN vector $n$ consists of $n_{R}$ independent noise components with $\operatorname{var}\left(\operatorname{Re}\left[n_{i}\right]\right)=\operatorname{var}\left(\operatorname{Im}\left[n_{i}\right]\right)=\sigma^{2} / 2$. Note that $\sigma^{2}$ represents both additive noise and interference from the surrounding cells. Hence, $\sigma^{2}$ is the interference-plusnoise power and is dependent on the cellular layout. The values of SINR $\Gamma$ described in Sec. II-A are related to $\sigma^{2}$ through the formula, $\sigma^{2}=n_{T} / \Gamma$. In this paper we do not consider overloaded systems. Thus, we have $n_{T} \leq n_{R}$.

If we have perfect CSI, we can perform a singular value

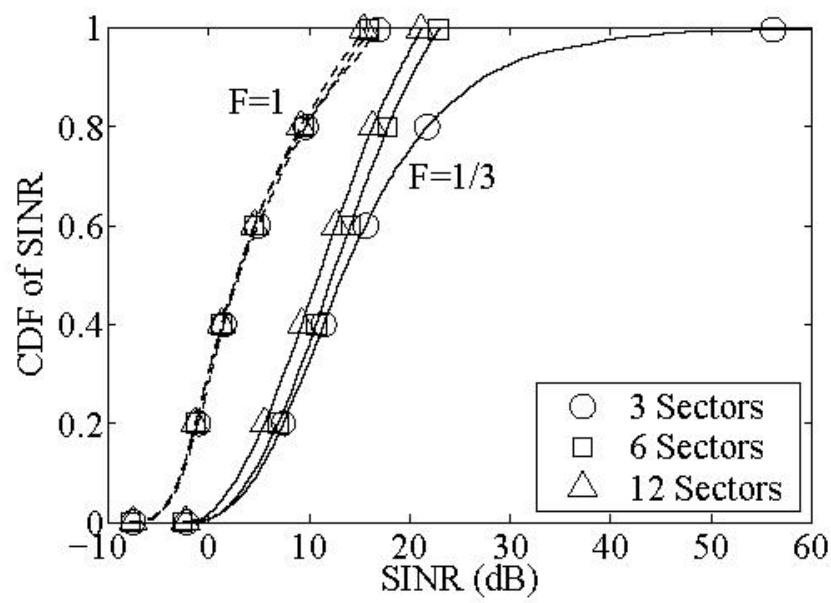

3: SINR CDF curves for all six cellular scenarios. decomposition of $H=U D V$, where $V$ is unitary and $U$ is the principal $n_{R} \times n_{T}$ submatrix of a unitary matrix. The matrix $D$ is diagonal with entries $\sqrt{\lambda_{k}}, k=1, \ldots, n_{T}$. Here, the $\lambda_{k}$ 's denote the distinct eigenvalues of $\boldsymbol{H}^{\dagger} \boldsymbol{H}$, where $(\cdot)^{\dagger}$ denotes the conjugate transpose.

As per conventional SVD-based spatial multiplexing methods [5], [0], we precode our $n_{T}$-dimensional symbol vector $b$ by multiplying it by $\boldsymbol{V}^{\dagger}$ and decode our received observation vector $r$ by multiplying it by $U^{\dagger}$. Defining $s=V^{\dagger} b$, $y=U^{\dagger} r$, and $\tilde{n}=U^{\dagger} n$ and transforming (1) by using the orthonormality of $U$ and $V$, the decoder output has the form

$$
y=D b+\widetilde{n} \text {. }
$$

Due to the orthonormality of $U^{\dagger}$, the transformed noise vector $\tilde{n}$ remains white Gaussian with $\operatorname{var}\left(\operatorname{Re}\left[\widetilde{n}_{\hat{q}}\right]\right)=\operatorname{var}\left(\operatorname{Im}\left[\widetilde{n}_{\hat{q}}\right]\right)=$ $\sigma^{2} / 2$. Because $D$ is diagonal, the MIMO channel in (1) has been transformed into $n_{T}$ parallel channels of the form

$$
y_{k}=\sqrt{\lambda_{k}} b_{k}+\widetilde{n}_{k}, \quad k=1, \ldots, n_{T} .
$$

Note that the SINR of the link, $\Gamma$, is given in Sec. IIA. With equal power allocation to the antennas, the SINR per eigenmode is $\Gamma / n_{T}$. The corresponding instantaneous SINR is defined by $\alpha_{k}=\lambda_{k} \Gamma / n_{T}$ for $k=1, \ldots, n_{T}$. The symbols $b_{k}$ are selected adaptively from various constellations according to the instantaneous SINR value, $\alpha_{k}$. The constellations considered are BPSK, 4-QAM and 16-QAM. In each case, the average symbol power is unity. Hence, for BPSK, $b_{k} \in\{-1,+1\}$, for 4-QAM, $b_{k} \in\{ \pm 1 \pm j\} / \sqrt{2}$ and for 16 QAM, $b_{k} \in\{(2 r-3)+j(2 s-3)\} / \sqrt{10}$, for $r, s \in\{0,1,2,3\}$.

These constellations are selected on the basis of an eigenvalue threshold vector $q=\left(q_{1}, q_{2}, q_{3}\right)$, where the entries of $q$ depend on $n_{T}$ and $\Gamma$. The exact dependence is not shown for ease of notation. When $\lambda_{k}<q_{1}$, the eigenmode is considered too weak for communication and transmission is suspended. When $q_{1} \leq \lambda_{k}<q_{2}$, BPSK is used. When $q_{2} \leq \lambda_{k}<q_{3}$, 4-QAM is used, and when $q_{3} \leq \lambda_{k}, 16$-QAM is used. The threshold vector, $q$, is selected on the basis of a target BER,

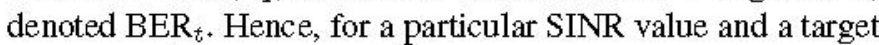
of $\mathrm{BER}_{t}$, the instantaneous BER of BPSK is $\mathrm{BER}_{t}$ when $\lambda_{k}=q_{1}$, the instantaneous BER of 4-QAM is BER $B_{t}$ when $\lambda_{k}=q_{2}$ and the instantaneous BER of 16-QAM is BER when $\lambda_{k}=q_{3}$. The target BER is therefore the maximum BER that the system experiences.

\section{THEORETICAL ANALYSIS}

\section{A. Bit Error Rates}

For a particular value of the SINR and $\lambda_{k}$, consider the received signal $y_{k}$ in (3). The BER values for the 3 different modulations can be approximated from the standard result, $\mathrm{BER} \geqq P$ (symbol error) $/ \log _{2} M$ where $M$ is the size of the constellation [7]. This gives the BER results [7]

$$
\begin{aligned}
P_{\varepsilon_{1} 1}(\lambda, \Gamma)= & 1-Q\left(-\sqrt{2 \alpha_{k}}\right) \quad \text { for BPSK } \\
P_{\varepsilon_{2}, 2}(\lambda, \Gamma)= & \left(1-Q^{2}\left(-\sqrt{\alpha_{k}}\right)\right) / 2 \text { for } 4 \text {-QAM } \\
P_{\varepsilon_{3}, 3}(\lambda, \Gamma)= & \frac{1}{4}-\frac{1}{16} Q(-\beta)-\frac{1}{16} Q(\beta)-\frac{3}{16} Q^{2}(-\beta) \\
& +\frac{1}{8} Q(\beta) Q(-\beta) \text { for } 16 \text {-QAM }
\end{aligned}
$$


where $\alpha_{k}=\lambda_{k} \Gamma / n_{T}, \beta=\sqrt{2 \alpha_{k} / 10}$ and $Q(\cdot)$ is the tail probability function for a standard Gaussian variable. Using the BER results in (4), the thresholds in $q$ can be computed by numerical solution of, for example, $P_{\varepsilon, 1}(\lambda, \Gamma)=q_{1}$.

\section{B. Eigenvalue Distributions}

The joint density of the unordered eigenvalues for an i.i.d. Rayleigh fading channel is well known [1] and is given by

$$
f_{\boldsymbol{\lambda}}(\boldsymbol{\lambda})=\frac{\Delta}{n_{T} !} \exp \left\{-\sum_{k=1}^{n_{T}} \lambda_{k}\right\} \prod_{k=1}^{n_{T}} \lambda_{k}^{n_{R}-n_{T}} \prod_{i<j}^{n_{T}}\left(\lambda_{i}-\lambda_{j}\right)^{2}
$$

with $\Delta=\left\{\prod_{k=1}^{n_{T}}\left[\left(n_{R}-k\right) !\left(n_{T}-k\right) !\right]\right\}^{-1}$. Integrating (5) over $\lambda_{2}, \ldots, \lambda_{n_{T}}$ and defining $\lambda=\lambda_{1}$ as an arbitrary eigenvalue leads to the density of $\lambda$ as [1]

$f(\lambda)=\sum_{i=1}^{n_{T}} \frac{(i-1) ! \lambda^{n_{R}-n_{T}}}{n_{T}\left(i-1+n_{R}-n_{T}\right) !} \exp (-\lambda)\left[L_{i-1}^{\left(n_{R}-n_{T}\right)}(\lambda)\right]^{2}$

where $L_{k}^{(n)}(\cdot)$ is a generalised Laguerre polynomial.

\section{The Error Free Rate}

In this paper we are interested in the performance of a particular type of adaptive scheme. Hence, we do not consider the channel capacity as in [3]. In assessing the performance of the scheme, the usual BER metric can be misleading due to the need to compare systems with different or variable modulations. Hence, we consider a metric which encapsulates both the BER and constellation size and is directly comparable across different systems. This metric is the error free rate, $R_{T}$, measured in bps/Hz, which can also be defined as the number of correctly received bits per symbol period. In the adaptive SVD scheme, $R_{T}$ can approach $4 n_{T}$ at high SINR, since each of the $n_{T}$ antennas can employ 16-QAM. Similarly, at low SINR, $R_{T}$ can approach zero when all eigenmodes suspend transmission. We focus on the mean value, $R=\mathrm{E}\left(R_{T}\right)$. Hence, $R_{T}$ is defined as the rate for a fixed SINR and $R$ is the overall rate, averaged over the SINR distribution.

For the single MIMO link in the desired cell, at a given SINR, the error free rate on the $i$-th eigenchannel is denoted $R_{i}$, and the total is $R_{T}=\sum_{i=1}^{n_{T}} R_{i}=n_{T} \bar{R}$, where $\bar{R}$ is the average rate. The overall rate is therefore defined by:

$$
R=n_{T} \mathrm{E}(\bar{R})=n_{T} \mathrm{E}\left(R_{o}\right)
$$

where $R_{0}$ is the error free rate of an arbitrary eigenmode. We denote an arbitrary eigenvalue by $\lambda$. The eigenvalue $\lambda$ is used to select the constellation, so that the probabilities of the constellations being adopted can be related to $\lambda$ as follows

$$
\begin{aligned}
P(\mathrm{BPSK}) & =p_{1}=P\left(q_{1} \leq \lambda<q_{2}\right) \\
P(4-\mathrm{QAM}) & =p_{2}=P\left(q_{2} \leq \lambda<q_{3}\right) \\
P(16-\mathrm{QAM}) & =p_{3}=P\left(q_{3} \leq \lambda\right) .
\end{aligned}
$$

The probabilities in (8) can be evaluated in closed form by direct integration of (6). This is conveniently performed in a symbolic manipulation package, such as Maple, although the exact results can also be written out with some extra effort. For reasons of space, these results are not presented here. Note that the values of $p_{1}, p_{2}$ and $p_{3}$ are also dependent on $n_{T}$ and $\Gamma$, but again this dependence is not shown.

For each constellation, a different number of bits per symbol are used ( 1 for BPSK, 2 for 4-QAM and 4 for 16-QAM), which are placed in the vector, $\boldsymbol{B}=\left(B_{1}, B_{2}, B_{3}\right)=(1,2,4)$. With this notation in place, we can derive $R$ as follows. First we compute $\mathrm{E}\left(R_{T} \mid \Gamma\right)$, the mean of $R_{T}$ for a given SINR. Then, we average over the SINR to obtain $R$ as follows

$$
\begin{aligned}
\mathrm{E}\left(R_{T} \mid \Gamma\right) & =n_{T} \mathrm{E}\left(R_{o} \mid \Gamma\right) \\
& =n_{T} \sum_{i=1}^{3} p_{i} \mathrm{E}\left(R_{o} \mid \Gamma \text {, constellation } i\right) \\
& =n_{T} \sum_{i=1}^{3} p_{i} B_{i}\left[1-\mathrm{E}\left(\mathrm{BER}_{o} \mid \Gamma \text {, constellation } i\right)\right]
\end{aligned}
$$

where $\mathrm{BER}_{0}$ is the instantaneous $\mathrm{BER}$ of an arbitrary eigenmode. Averaging over $\mathrm{BER}_{0}$ allows (9) to be rewritten as

$$
\mathrm{E}\left(R_{T} \mid \Gamma\right)=n_{T} \sum_{i=1}^{3} p_{i} B_{i}\left[1-\int_{q_{i}}^{q_{i+1}} P_{\varepsilon, i}(\lambda, \Gamma) f_{i}(\lambda) d \lambda\right]
$$

where $f_{i}(\lambda)$ is the density of $\lambda$ conditioned on constellation $i$ being used. Hence, $f_{i}(\lambda)=f(\lambda) / p_{i}$ for $q_{i} \leq \lambda<q_{i+1}$, $i=1,2,3$ and $q_{4}=\infty$. Substituting $f_{i}(\lambda)$ into $(10)$ gives

$E\left(R_{T} \mid \Gamma\right)=n_{T} \sum_{i=1}^{3} B_{i}\left[p_{i}(\Gamma)-\int_{q_{i}(\Gamma)}^{q_{i+1}(\Gamma)} P_{\varepsilon, i}(\lambda, \Gamma) f(\lambda) d \lambda\right]$

where the dependence of $p_{1}, p_{2}, p_{3}$ and $q_{1}, q_{2}, q_{3}$ on the value of $\Gamma$ is now explicitly shown. Averaging (11) over $N_{\text {SINR. }}$ simulated values of the SINR gives

$$
\begin{aligned}
R= & \frac{1}{N_{S I N R}} \sum_{k=1}^{N_{\text {SINR }}} n_{T} \sum_{i=1}^{3} B_{i} \\
& \times\left[p_{i}\left(\Gamma_{k}\right)-\int_{q_{i}\left(\Gamma_{k}\right)}^{q_{i+1}\left(\Gamma_{k}\right)} P_{\varepsilon, i}\left(\lambda, \Gamma_{k}\right) f(\lambda) d \lambda\right] .
\end{aligned}
$$

The finite range integral in (12) has to be computed numerically, although we note that if the range was $[0, \infty)$ then standard methods [8] could be used. In all our calculations, $N_{\text {SINR }}=500$ was used. Increasing $N_{\text {SINR }}$ to 5000 was also tested and results were found to be only marginally different.

\section{SiMULATION RESULTS}

Figure 4 shows the error free rate, $R_{T}$, for the MIMO SVD scheme, with a target BER of $10^{-1}$. Results are shown for a range of fixed SINR values from $-5 \mathrm{~dB}$ to $15 \mathrm{~dB}$ for $(1,1)$, $(2,2),(4,2)$ and $(4,4)$ schemes. The lines indicate analytical calculations obtained from (11). The points represent simulation results. Note the slight difference between the simulations and analysis due to the use of the BER approximation in (4) which leads to the analytical calculations being marginally below the simulated results. Figure 4 is a useful verification of the analysis method and clearly shows the massive drop in $R$ as the SINR is reduced, especially for larger systems. 
Inspection of the CDFs in Fig. 3 shows that the SINR distributions vary in spread as well as in mean. As the mean SINR is increased, the performance will increase, but it is less clear how the variance will affect the system. Hence, we consider an underlying Gaussian SINR distribution, with mean value equal to the mean SINR of the R1S3 scenario and a variance which ranges from zero to roughly double the actual variance of the R1S3 data. Note that the Gaussian distribution gives a simple approach to investigating the effect of variance, and is not a realistic model for the SINR. In Fig. 5 we plot $R$ vs. the variance for a target BER of $10^{-3}$ and varying system sizes. Clearly, increasing the variance improves performance, and this is most noticeable for larger systems. Since the $F=1 / 3$ CDFs show greater variance than the $F=1$ case, increasing the variance of the SINR will have similar effects to introducing frequency reuse, which results in reduced intercell interference. Hence, all the systems considered will benefit from this. Figure 5 also allows a comparison of the effects of system size. MIMO systems inherently have spatial selfinterference. Therefore, at the receiver, the degrees of freedom available are partly consumed in removing self-interference. A comparison of the $(2,2)$ and $(2,4)$ system results show that the presence of extra receive diversity in the $(2,4)$ system provides additional degrees of freedom that is helpful in mitigating the effects of inter-cell interference. Hence, the superior performance of the $(2,4)$ system. Figure 6 focusses on a $(4,4)$ system and shows the impact of SINR variance for different target BERs. The effect of the variance is shown to increase as the target BER is lowered.

Figure 7 is a central result and shows the trade off between BER and $R$ for a $(4,4)$ system. For target BERs down at $10^{-4}$, the values of $R$ are generally less than half the values at a target of $10^{-1}$. Clearly, with the cellular SINR distributions considered, the overall error free rate is far from the maximum of 16. At acceptable BER values, even the best case only gives values around 6-7, and the worst case is between 1 and 2 .

Next we consider slight variations in the adaptive SVD scheme. In particular, we remove the 16-QAM option or the suspended transmission mode. If the suspended transmission mode is removed, then transmission always occurs, even on the worst eigenmode. Figure 8 shows a comparison of the performance of the standard scheme with these 2 variations. The effect of removing the 16-QAM option is very large, and the values of $R$ drop markedly. Clearly, it is very important to be able to use the highest modulation schemes for periods of large SINR. Removing the suspended transmission mode increases $R$, but the change is not so dramatic and brings with it increased BER.

The results to date show that the adaptive scheme performs far below the maximum possible. Hence, it is important to compare the adaptive scheme with other detection methods. Therefore, we also consider MIMO-MMSE combiners operating with fixed BPSK or 4-QAM modulations. Details can be found in [9]. All system sizes from $(1,1)$ to $(4,4)$ were simulated, and for each system both $R$ and the average BER, averaged over all SINR values, were simulated. In addition, the adaptive SVD was considered over a range of target BERs. At each target BER, the average BER was evaluated by simulation and $R$ was obtained from (12). Results are shown in Fig. 9. In comparison to the fixed transmission scheme, the adaptive scheme performs far better. Its ability to use higher-order modulations at high SINR and reduce the constellation size or suspend transmission during very low SINR periods allows it to maintain reasonable values of $R$ at average BER values, well below those offered by the MIMO-MMSE receivers.

Figures 10 and 11 illustrate the behaviour of the probability that transmission is completely suspended on all eigenchannels, $P_{S}$. This can be obtained from $P_{S}=P\left(\lambda_{\max }<q_{1}\right)$, and these can be computed analytically by integrating (5). Figure 10 shows how $P_{S}$ drops with SINR for various system sizes using a target BER of $10^{-3}$. The $(1,1)$ system requires large SINR values around $20 \mathrm{~dB}$ before $P_{S}$ approaches zero. The $(4,4)$ system is best, but still requires around $5 \mathrm{~dB}$, and inspection of the CDFs for $F=1$ shows that this SINR is available with a probability less than 0.5 . Figure 11 shows how the target BER affects $P_{S}$ for a $(4,4)$ system. Target BERs of $10^{-2}, 10^{-3}$ and $10^{-4}$ all require an SINR around $5 \mathrm{~dB}$ for $P_{S}$ to approach zero. Hence, the BER vs. $R$ trade off provided by the adaptive scheme is created at the expense of a high value of $P_{S}$ at SINR values below $5 \mathrm{~dB}$.

In Figs. 12 and 13 we consider the comparative performance of diversity vs. MIMO and adaptive vs. fixed modulations. Figure 12 shows the error free rates achieved by various diversity schemes with the $(4,4)$ system also shown for comparison. All systems use the standard adaptive approach, employing 16QAM, 4-QAM, BPSK or transmission suspension, depending on the channel. At all target BERs increased diversity yields improved rates, and at low target BERs the $(1,4)$ scheme is only marginally worse than the $(4,4)$ system. Figure 13 shows the impact of adaptive modulation. The bottom 3 curves are for a scheme where transmission is either suspended or a fixed 16-QAM, 4-QAM or BPSK modulation is employed. In the legend, such a scheme is labelled as "Fixed". The standard adaptive scheme for $(1,4)$ and $(4,4)$ systems are also shown for comparison. At low target BERs the adaptive $(1,4)$ scheme offers more than a $60 \%$ improvement over the fixed modulations. The improvement over the $(1,4)$ diversity approach offered by the $(4,4)$ system is less than $30 \%$.

\section{CONCLUSION}

In this paper, we have derived and verified the error free rate of an adaptive MIMO SVD system. This performance metric is useful in comparing both fixed and adaptive transmission schemes with varying constellation sizes. We show the effect of the cellular environment on performance, specifically the effects of cellular layout and the variance of the SINR distribution. Key results are presented concerning the effect of adaptive modulation and the performance gap between diversity methods and MIMO. In particular, we show that the improvement offered by the adaptive SVD approach is at the expense of suspending transmission, and these periods of no communication occur with high probability at SINR values below $5 \mathrm{~dB}$. For the cellular layouts considered, these SINR values are extremely common. Finally, we demonstrate 


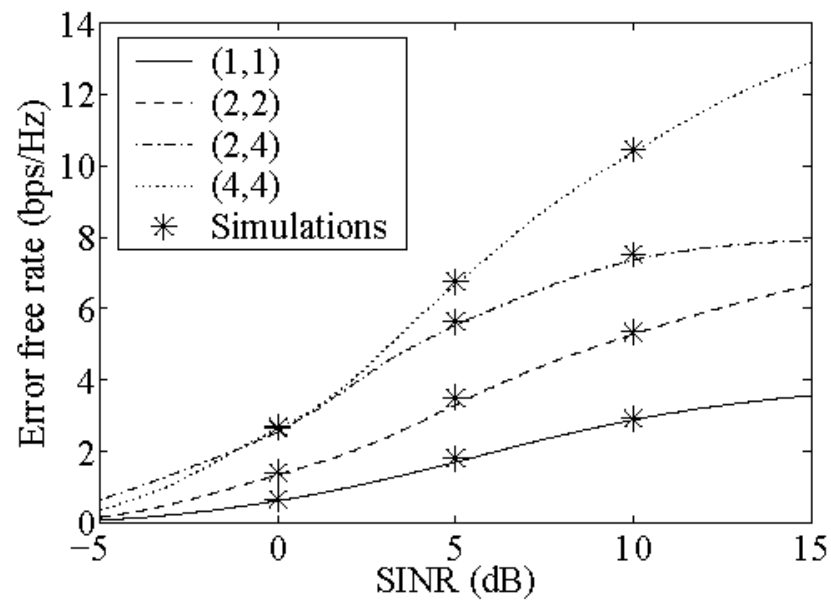

4: Error free rate Vs. SINR for $(1,1),(2,2),(2,4)$ and $(4,4)$ systems, with a target BER of $10^{-1}$.

that the improvement of the $(4,4)$ adaptive SVD scheme over the $(1,4)$ diversity scheme is relatively small, considering the complexity increase and feedback requirements. We conclude that in the cellular environments considered, relative to a SISO system, the majority of the performance gain due to the $(4,4)$ system can be obtained via an adaptive diversity system.

\section{ACKNOWLEDGEMENT}

The authors wish to thank Howard Huang of Lucent Technologies for supplying the simulated cellular SINR data.

\section{REFERENCES}

[1] I. E. Telatar, "Capacity of multi-antenna Gaussian channels," European Trans. on Telecomm. Related Technol, vol, 10, pp. 585-595, Nov.-Dec. 1999 .

[2] G. J. Foschini and M. J. Gans, "On limits of wireless communication in a fading environment when using multiple antennas," Wireless Personal Commin, vol. 6, no. 3, pp. 311-335, Mar. 1998.

[3] H. Huang and R. Valenzuela, "Fundamental simulated performance of downlink fixed wireless cellular networks with multiple antennas" in Proc. IEEE Int'l. Symp, on Personal and Mobile Radio Communications, Berlin, Germany, Sept. 11-14, 2005.

[4] M. Grant, "Draft report on radio aspects for the terrestrial component of IMT-2000 and systems beyond IMT-2000," ITU Document 8F/TEMP/216 (Rev. 3), Attachment 6.9, Sept. 30, 2005.

[5] G. G. Raleigh and J, M. Cioff, "Spatio-temporal coding for wireless communication," IEEE Trans. Commun, vol, 46, no. 3, pp. 357-366, Mar. 1998.

[6] G. Lebrun, T. Ying, and M. Faulkner, "MIMO transmission over a timevarying channel using SVD," in Proc. 2002 IEEE Global Telecommunications Conf., Taipei, Taiwan, Nov, 17-21, 2002, pp. 414-418.

[7] J. G. Proakis, Digital Communications, 4th ed. New York: McGraw-Hill, 2001.

[8] M. K. Simon and M.-S. Alouini, Digital Communication over Fading Channels: A Unified Appraach to Performance Anabysis. New York John Wiley \& Sons, 2000.

[9] L. M. Garth, P. J. Smith, and M. Shafi, "Exact symbol error probabilities for SVD transmission of BPSK data over fading channels," in Proc. 2005 IEEE Int'l Conf. on Communications, Seoul, Korea, May 16-20, 2005, pp. 2271-2276.

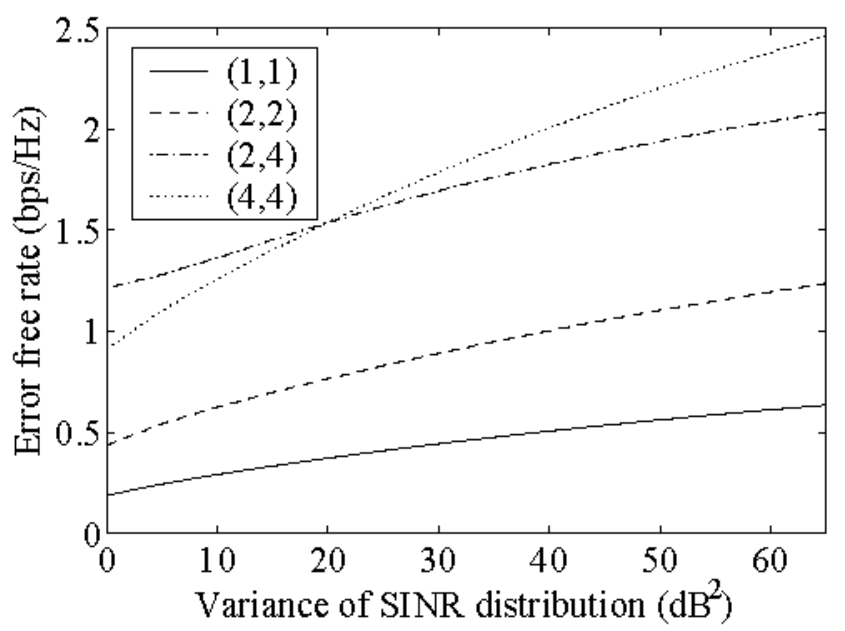

5: Error free rate vs. variance of the SINR distribution for $(1,1),(2,2),(2,4)$ and $(4,4)$ systems, with a target BER of $10^{-3}$.

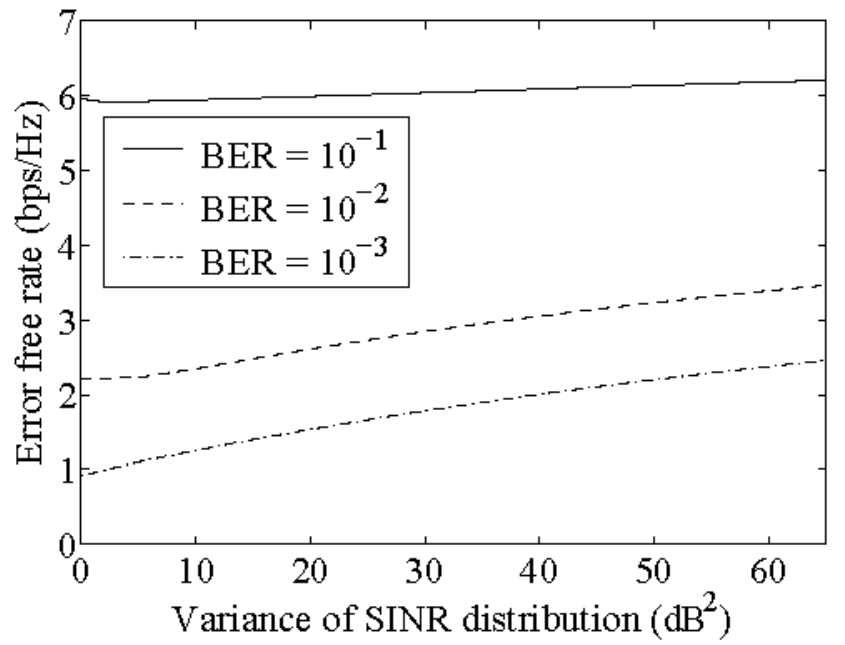

6: Error free rate vs, variance of the SINR distribution for a $(4,4)$ system, with target BERs of $10^{-1}, 10^{-2}$ and $10^{-3}$.

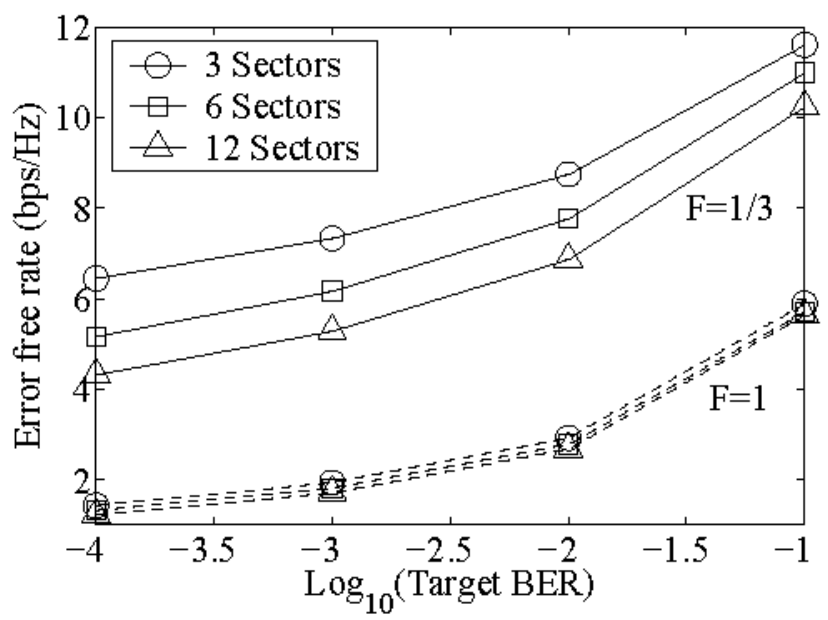

7: Error free rate vs, target BER for all six cellular scenarios for a $(4,4)$ system. 


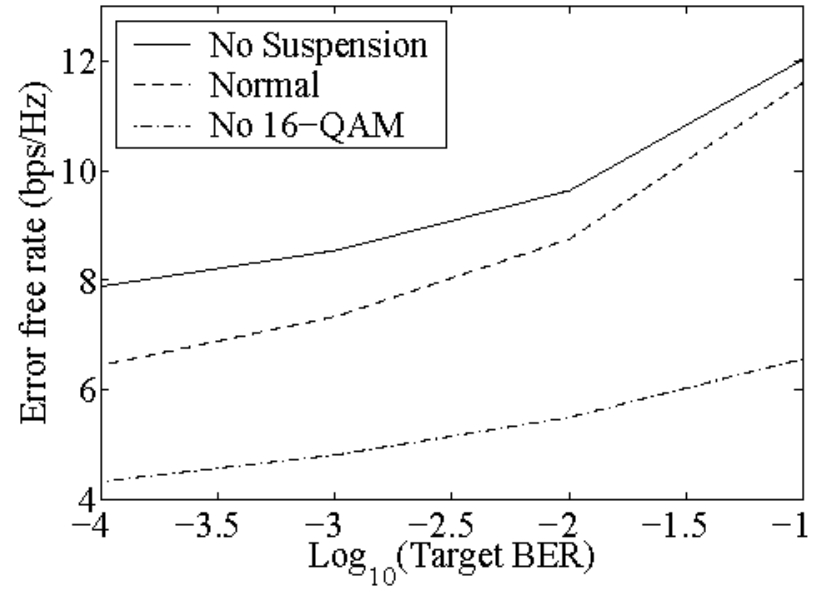

8: Error free rate vs, target BER for cellular scenario R3S3, with three versions of adaptive transmission for a $(4,4)$ system.

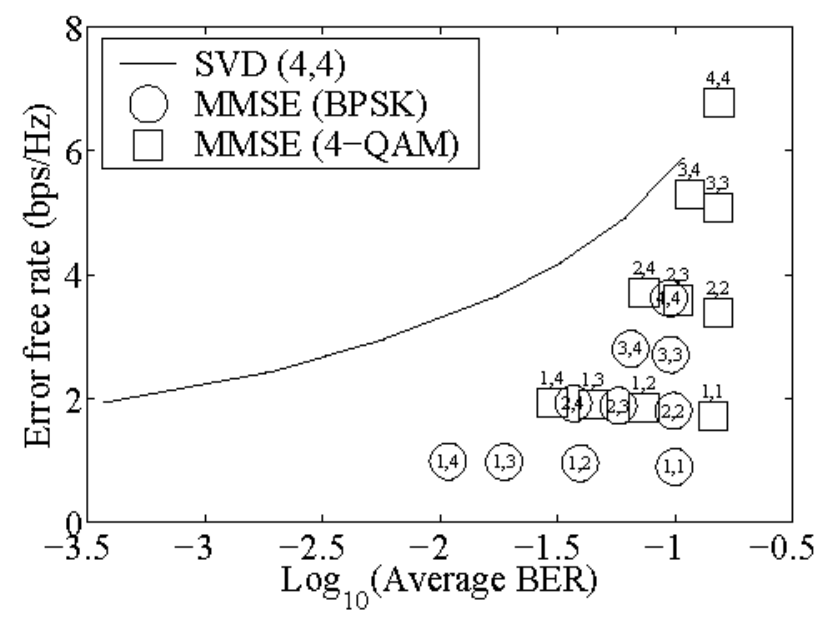

9: Error free rate vs, average BER for SVD adaptive transmission and MIMO-MMSE linear combiners, for cellular scenario R1S3.

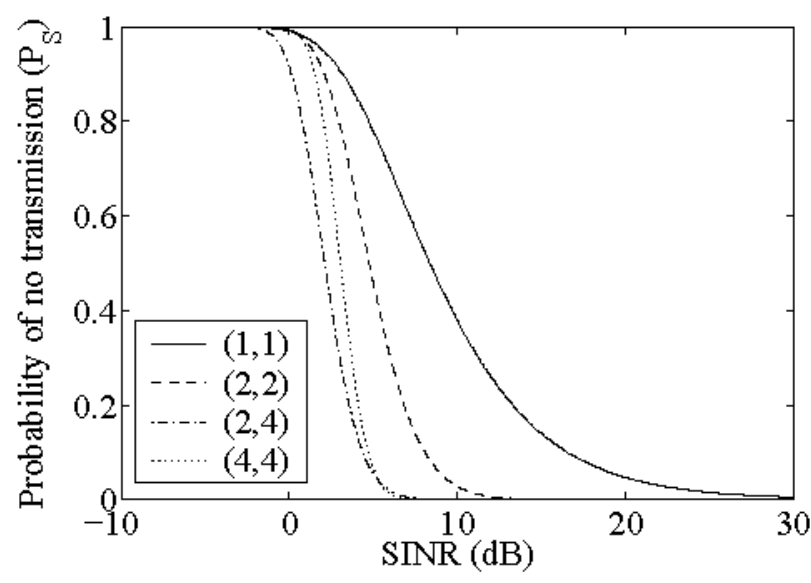

10: Probability of no transmission vs. SINR for $(1,1),(2,2),(2,4)$ and $(4,4)$ systems, with a target BER of $10^{-3}$.

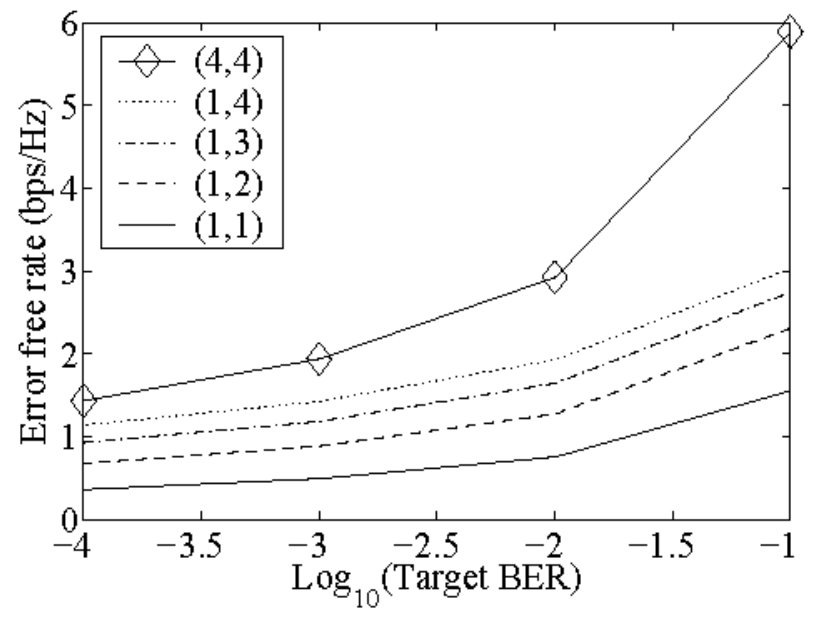

12: Error free rate vs, target BER: effect of RX diversity on SVD adaptive transmission for cellular scenario R1S3.

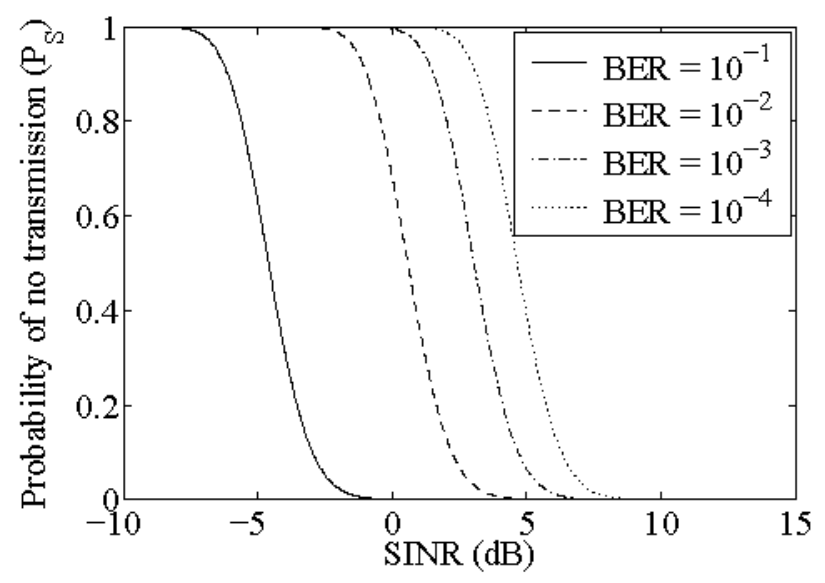

11: Probability of no transmission vs. SNR for a $(4,4)$ system with tar get BERs of $10^{-1}, 10^{-2}, 10^{-3}$ and $10^{-4}$.

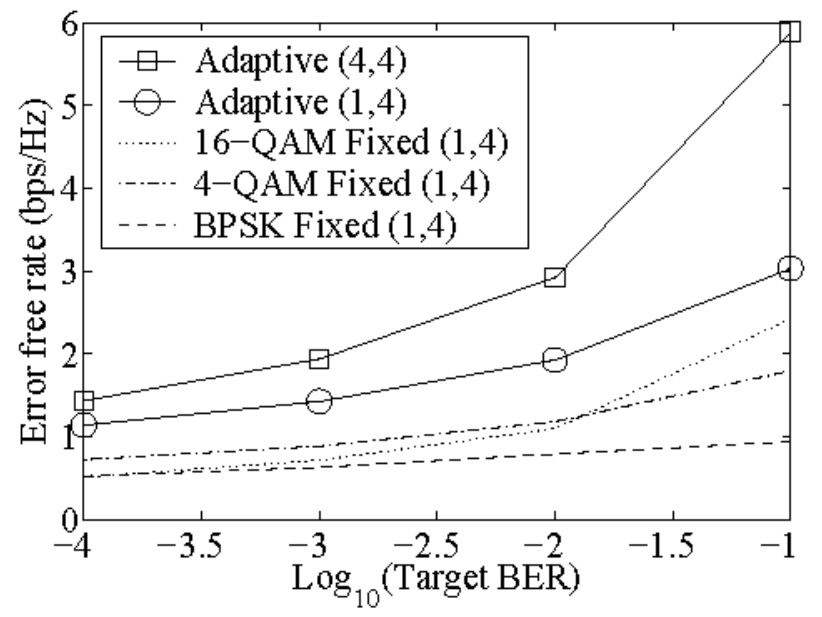

13: Error free rate vs, target BER: adaptive vs, fixed SVD modulation schemes for cellular scenario R1S3. 